\title{
In Ice Neutrino Radio Detection Projects
}

\author{
Allan Hallgren ${ }^{1}$ \\ Department of Physics and Astronomy, Uppsala University \\ Box 516, 75120 Uppsala, Sweden \\ E-mail: allan.hallgren@physics.uu.se
}

Ultra high energy neutrinos are promising messengers from the most energetic processes in the universe. Measurements of spectral and directional properties of a sample of such neutrinos will provide important clues for the understanding of the origin of the highest energy cosmic rays, detected at $\sim 10^{20} \mathrm{eV}$. Simultaneously neutrino and fundamental physics at otherwise unreachable energies can be investigated. The detection of a sufficient number of these neutrinos requires monitoring large target masses. Such measurements are likely best and most cost effectively achieved with in-ice radio-frequency pulse detection systems. This contribution gives an overview of the method followed by a description of the first experiment of this kind, RICE and of currently planned systems. The three projects for in-ice radio detection of neutrinos, ARA, ARIANNA and GNO are described. Results from prototype test stations of ARA and ARIANNA are promising. The path towards large scale systems should be followed.

XVI International Workshop on Neutrino Telescopes

2-6 March 2015

Palazzo Franchetti - Istituto Veneto, Venice, Italy

\section{${ }^{1}$ Speaker}




\section{Introduction}

Cosmic rays were discovered by Victor Hess more than 100 years ago. Since then much has been learned about their properties but many important questions remain un-answered. This is in particular true for the high energy end of the observed spectrum, reaching energies as high as $10^{20} \mathrm{eV}$. Large detector arrays are operating and provide an increasing amount of data, but the locations and acceleration mechanism behind these extremely high energy particles remain enigmatic.

Neutrinos have been proposed as unique messengers that would provide information on the origin and production of cosmic rays. Being electrically neutral the neutrino trajectories will not be bent by the galactic and inter-galactic magnetic fields, their direction will point back directly to their production site. Being subject only to the weak interaction they further can escape from denser regions where acceleration of hadrons may occur and then propagate unhindered over long distances. This is in contrast to the highest energy cosmic rays whose range is limited by interactions with matter and in particular by the background photon field. Neutrinos are, for the same reason, very difficult to detect. Their low cross-section necessitates use of large target masses for the detectors and several projects have been proposed, some of which have been built. Most of these intend to observe Cherenkov radiation from charged particles produced in neutrino interactions, thus optical transparency in large masses is important. Detectors of this type will use the atmosphere, sea-water or glacial ice.

The first detection of high energy neutrinos of cosmic origin was recently accomplished by the IceCube collaboration, operating a detector system with over 5000 optical modules installed in one cubic kilometer of deep glacial ice at the geographic South Pole. The few tenths of cosmic neutrinos detected reach energies up to a few PeV [1][2]. This detection constitutes the first step in high energy neutrino astronomy. The spectrum and directional distribution of these neutrinos is being analyzed from many aspects in a large number of articles. The sample will grow with observation time, providing for improved spectral and compositional information, possibly directional clustering will make association to known astrophysical objects possible. Plans for expanding IceCube to a next generation detector with about 10 times larger instrumented volume are under development, and if funding is granted construction can start in a few years [3].

To detect a reasonable number of even higher energy neutrinos of cosmic origin, with energies of about $1 \mathrm{EeV}\left(10^{18} \mathrm{eV}\right)$ corresponding to the flux of the highest energy cosmic rays it is though necessary to build detectors observing much larger target masses. The optical method used in IceCube will then be prohibitively expensive. Fortunately other detection mechanisms become possible at these energies, in particular detection of radio signals from intense electromagnetic showers developing in radio-transparent ice. To reach the lowest thresholds possible the receiver antennas should be embedded in the ice ${ }^{2}$. This presentation will give an overview of the method and the current status of the projects now being developed.

\footnotetext{
${ }^{2}$ Methods to lower the threshold for balloon-borne antennas are under development, but will not be covered in this contribution.
} 

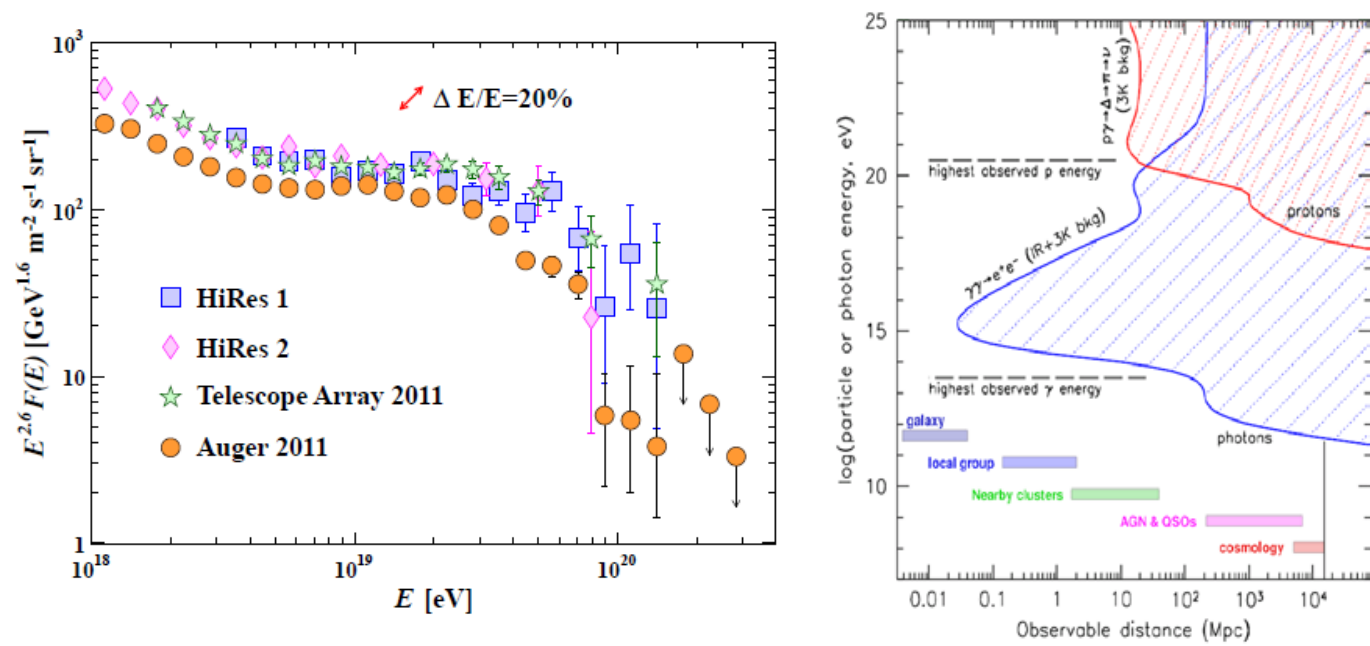

Fig. 1. Left: The spectrum of cosmic rays in the energy range above $10^{18} \mathrm{eV}$. Note multiplication by the factor $E^{2.6}$. A cut-off at $\sim 10^{19.5} \mathrm{eV}$ is visible. From [4]. Right: Propagation range in the universe for $\gamma$ and protons. High energy protons interact with CMBR, limiting propagation to $\sim 50 M p c$.

\section{Ultra High Energy Neutrino Flux Predictions}

The spectrum of cosmic rays has been measured over a wide range of energies, up to energies of $\sim 10^{20} \mathrm{eV}$ where the flux is $<1$ particle $/ \mathrm{km}^{2} /$ year. The upper part of this spectrum is shown in Fig. 1. For the highest energies the propagation of charged particles through the universe is limited by interactions with photons in the cosmic microwave background radiation (CMBR). At these energies, $\gtrsim 5 * 10^{19} \mathrm{eV}$, protons interacting with the photons produce $\Delta$ resonances that decay to pions and subsequently yield neutrinos. These neutrinos are dubbed GZK-neutrinos after Greisen, Zatsepin and Kuzmin who proposed this mechanism [5]. The limited range for charged particle propagation is illustrated in fig. 1, where it can be seen that e.g. protons with energy of $10^{20} \mathrm{eV}$ will not reach us from beyond $\sim 50 \mathrm{Mpc}$, within our local supercluster.

The neutrino flux from the GZK mechanism has not yet been detected but it is considered to be "guaranteed", fig 2. The flux level and spectral distribution of the GZK neutrinos is though dependent on several factors. If the nuclear composition of the cosmic rays at high energies has a high fraction of heavy elements then the GZK neutrino flux will be reduced. Also the cosmic evolution of the sources influence the flux due to that the CMBR photons are more energetic at higher redshifts. A lower energy part of the spectrum is predicted from decay of neutrons. Several predictions for the GZK flux have been made with variations of the above and other factors, resulting in large variations of the predicted flux. For this reason a measurement of the flux level and spectral distribution of GZK neutrinos will provide valuable information on the contributing factors. The more optimistic flux models have already been disfavored by experimental results, the situation is summarized in fig. 4.

Directional clustering of GZK neutrinos or other association to specific astrophysical objects will provide information on sites for generation of the highest energy cosmic rays, throughout a large part of the universe. In addition to cosmic ray related physics the distribution of events in a region around the horizon will provide information on the cross section for neutrinos at energies that cannot be studied elsewhere, and may allow conclusions on 'new physics' [6] as illustrated in fig 2. 

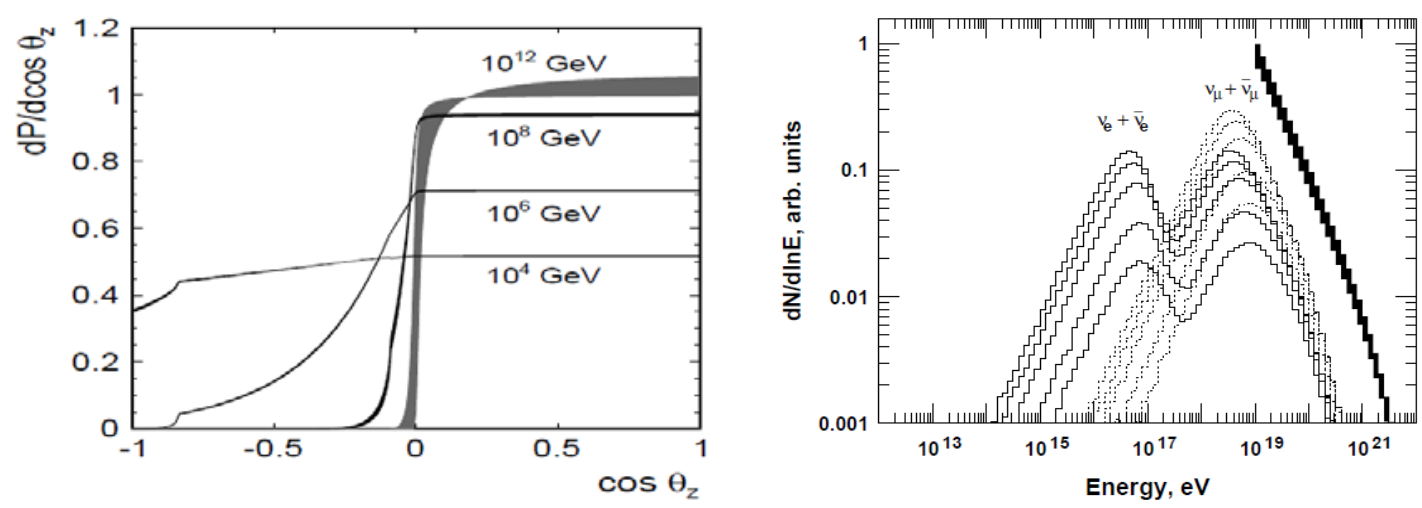

Fig. 2. Left: The distribution of events just below the horizon provides information on the energy dependence of the neutrino cross-section, the figure illustrates a possible scenario. From[6].

Right: Predictions for neutrino spectrum from GZK mechanism, after propagation over 10, 20, 50, 100, $200 \mathrm{Mpc}$ (from bottom up). The rightmost distribution corresponds the proton injection spectrum, the peak to the right is from $\pi$ and $K$ decay, the peak to the left is from possible neutron decay [7]. The radio detection projects focus on the right peak.

From the predicted fluxes it is clear that large detectors will be needed to obtain a useful sample of events in a reasonable time period. Estimates show that target masses corresponding to several $100 \mathrm{~km}^{3}$ of ice are needed. The most cost effective detection system proposed is based on detection of radio pulses from neutrino-induced electromagnetic showers.

\section{Askaryan Radio Pulse Generation and Properties}

Radio detection of neutrinos builds on a mechanism proposed by G. Askaryan [8]. Particle showers initiated by an interacting neutrino will due to the electrons in the medium develop an negative charge excess of $\sim 20 \%$ traveling through the medium as a compact bunch at speed c. Cherenkov-type radiation is then generated which for wavelengths corresponding to the transverse size of the electron bunch adds coherently and radio-frequency signals are generated. The power of the radiation depends on the number of electrons squared, thus on the shower energy squared. The wavelengths depend on the lateral size of the shower, and will typically be $\gtrsim$ few dm. A short bipolar pulse will result. The effect has been calculated and Monte-Carlo simulated. As showers of individual events develop differently the generated radiation will show some variability in e.g. angular spread vs frequency, but in a way that can be modelled [9].

Radio frequency emission by the Askayan effect has been verified experimentally in ice and in sand. A test-beam at SLAC with $\sim 10^{9}$ electrons at $28.5 \mathrm{GeV}$ was dumped in the target material to create high energy showers and the RF radiation was measured [10]. The power, frequency content and angular distribution were found to be in good agreement with the theory.

The medium has to be radio-transparent in order to be of interest for a detector. Ice has a long attenuation length for RF radiation. Other considered media are dry sand or large salt formations. The ANITA project has flown balloon-born antennas over Antarctica to look for neutrino-induced signals. No signals were yet attributed to neutrinos, but data from the last campaign (ANITA-III) are still being analyzed. Large radio telescopes have looked for Askaryan pulses from the regolith of the Moon. Also these attempts have yet not detected pulses from neutrinos. A drawback with having the detector placed at large distance from the signal generation is that the threshold goes up. To reduce the threshold the receivers should be placed close to the interaction target, as is the case for the projects RICE, ARA ARIANNA and GNO that will be described below. 

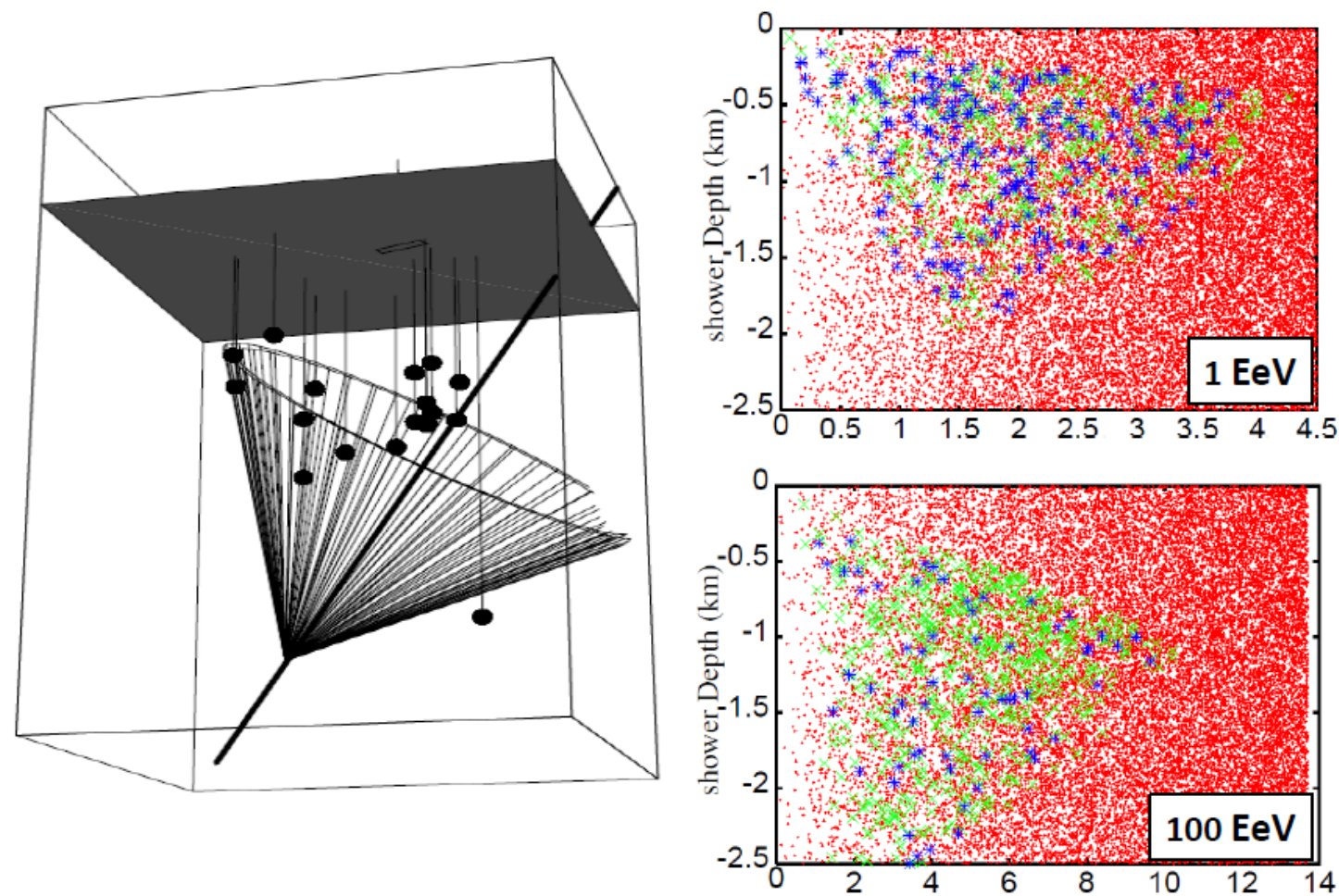

Fig. 3. Left: Illustration of the RICE detector and a simulated neutrino event. The black dots correspond to the radio recievers placed in holes in the ice, hanging down from the surface of the snow (grey plane). The neutrino direction is indicated with the diagonal line and the RF radiation is shown with the lamp-shade shape [11]. Right: Simulated radial and depth distribution of neutrino interactions in the ice, at two different energies. The red points are undetected interactions, the blue and green are detected EM and hadron induced showers respectively. The hadronic part takes over at the higher energy. Note the difference in the radial distance scale (in $\mathrm{km}$ ) between the energies[12].

\section{The Pioneering Radio Ice Cherenkov Experiment RICE}

The first experiment with an array of recievers installed in ice was the Radio Ice Cherenkov Experiment (RICE) that took advantage of the construction of the optical neutrino telescope AMANDA at the geographic South Pole [11]. The RICE array had 20 recievers installed at depths $100-200 \mathrm{~m}$ below surface, one was installed at $350 \mathrm{~m}$ depth. Typical horizontal distances were about $200 \mathrm{~m}$ in each direction. A sketch of the detector and a simulated event is seen in fig. 3. Noise from the photomultipliers of the AMANDA array (straight below, at depths 1450-2000 m) was reduced with a filter removing frequencies $<200 \mathrm{MHz}$.

The result of simulations at two different energies is shown in fig. 3. As the RF radiation is emitted in a limited angular region the direction of the incoming neutrino and the interaction point has to combine favorably to generate signals in the receivers. Therefore undetected events are mixed with detected events throughout the volume, as seen in fig. 3. The simulations included pure electro-magnetic showers and hadron induced showers. The LPM-effect (Landau, Pomerancuk, Migdal) influence the longitudinal development of the shower [9] and was included in the simulations. The generation of signal was simulated with charge-by-charge superposition of Cherenkov radio frequency emission. The simulation allowed studying effects from refraction, attenuation, gain variations, modifications of antenna response in changing medium from ice/firn to air. It is worth noting that at the higher neutrino energies of $100 \mathrm{EeV}$ the detection is dominated by hadronically induced showers while at $1 \mathrm{EeV}$ the direct EM shower component is dominating for the detection. 


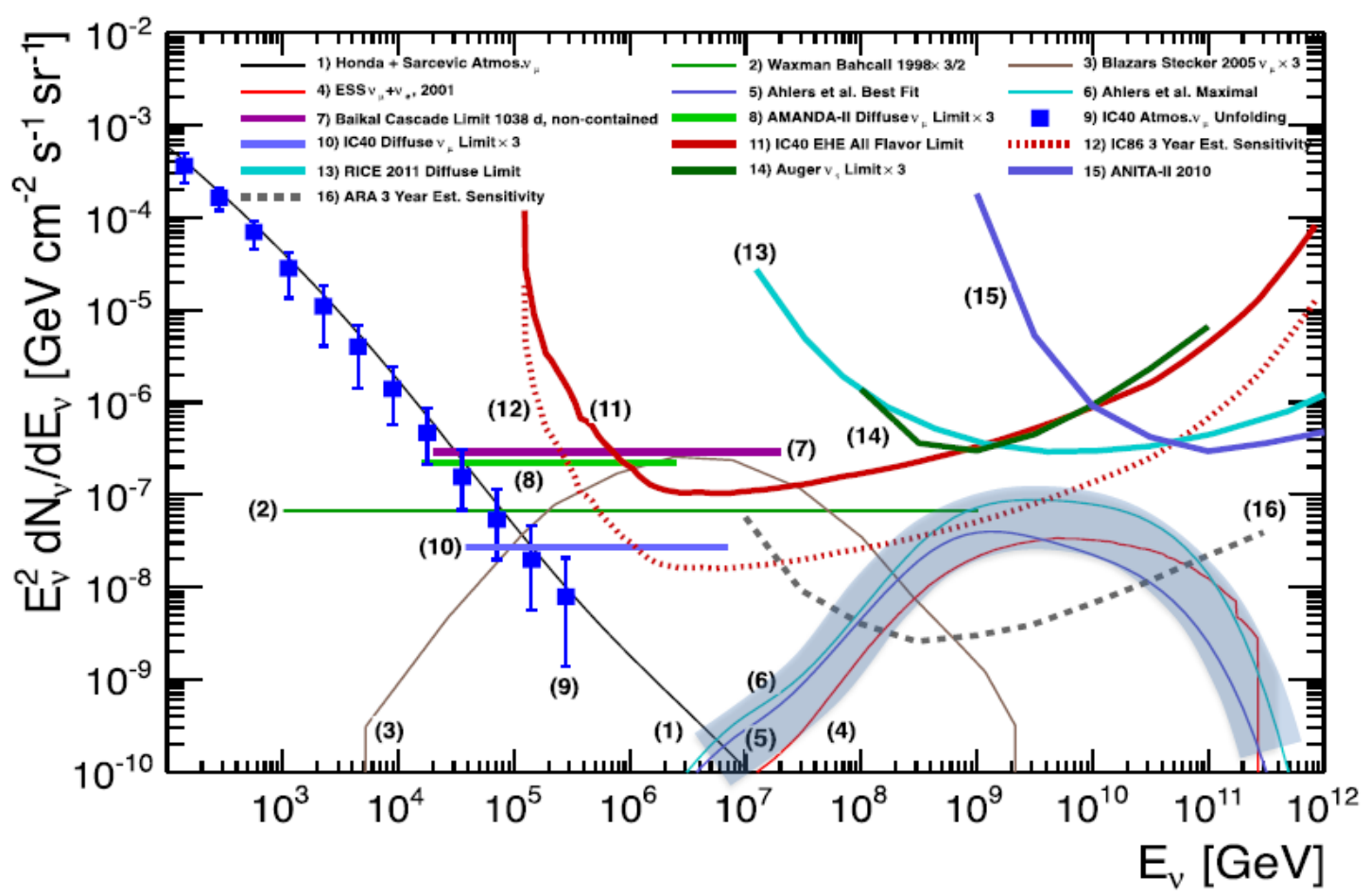

Fig. 4. The neutrino spectrum, limits and models. Most relevant for in ice radio detection of neutrinos is the limit from RICE ([13], turquoise line), the ARA sensitivity ([14], dashed line)and the band of popular GZK-neutrino predictions (shaded light blue). See also fig 7. The astrophysical flux of neutrinos recently measured by the IceCube collaboration in the energy range up to a few PeV is not included in this figure.

Data were collected during 1999 - 2010, for a total of 1700 live-days. The analysis removed noise due to radio transmitters at the surface, true thermal noise and the remaining PMT induced interference that passed the $200 \mathrm{MHz}$ filter. Timing of the signals on the different antennas allows reconstructing the event vertex position and events close to the surface were removed. No events remained after all cuts, resulting in a competitive limit [13].

\section{Present development projects}

\subsection{ARA}

The Askaryan Radio Array (ARA) project intends to instrument the ice close to the IceCube detector at the South Pole. After some tests with equipment installed in the upper part of holes drilled for IceCube a small “Testbed”-system was installed in December 2010 at 20-30 $\mathrm{m}$ depth in a location $\sim 1 \mathrm{~km}$ off to the side of IceCube [14]. The following seasons three more stations were installed, the last two (ARA2, ARA3) in 2012-2013 reached full design depth of $\sim 200 \mathrm{~m}$. The station spacing is $2 \mathrm{~km}$ and the planned number of stations is 37, see fig. 5 . The array of the three stations has taken calibration and physics data. A first publication from the three stations is in preparation.

Each station has 4 holes for detectors and 2 holes for calibration equipment. Four receiver antennas are placed in each of the four holes, with some vertical separation. Two of the antennas are horizontally polarized, two vertically polarized. The design of the antennas is strongly constrained by the need to fit into the fairly narrow holes that have a diameter of $15 \mathrm{~cm}$. The frequency range covered is $\sim 200-850 \mathrm{MHz}$; a notch filter has been included in the electronics to reduce interference from communication radio at the South Pole station. 


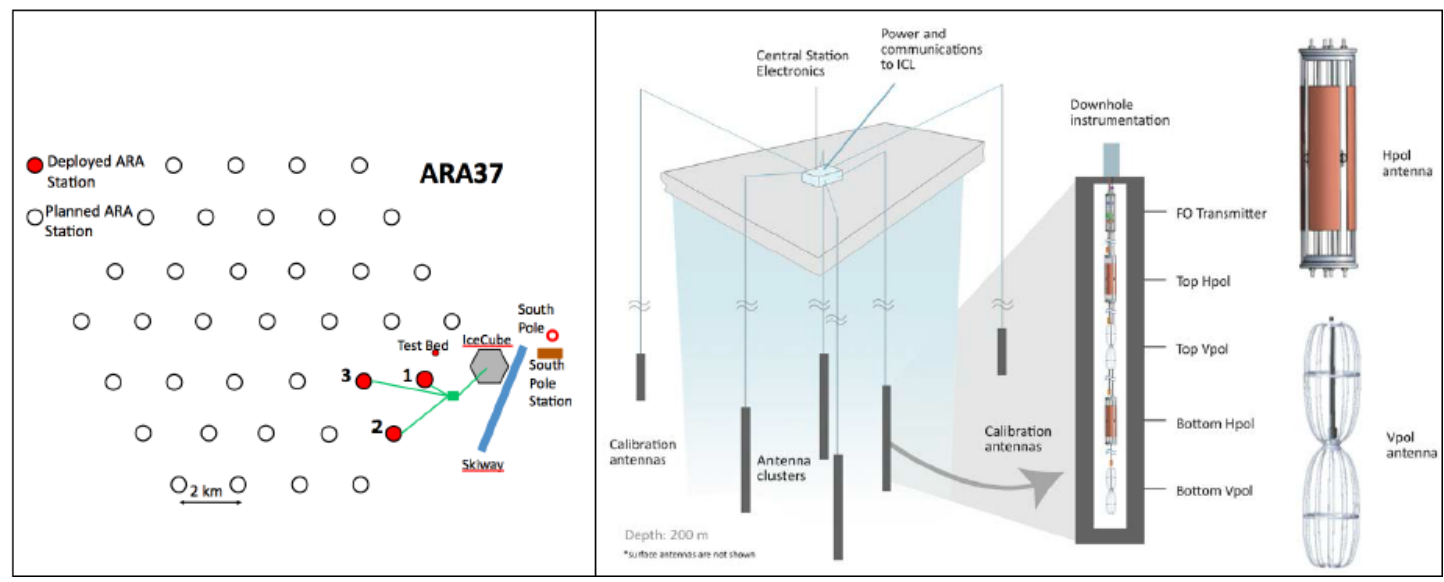

Fig. 5. The ARA project. Left: Shows the proposed layout of the proposed full ARA system with 37 stations. The inter-station spacing is $2 \mathrm{~km}$. The positions of the three first stations is indicated with red filled circles. Other objects indicated is the foot-print of the IceCube detector, the South Pole station main building, the Pole marker and the landing strip. Right: Schematic of one station, with 4 holes for detector antennas drilled to $200 \mathrm{~m}$ depth and two holes for calibration transmsitters. A detail shows the positions of four antennas in each of the four holes, with a vertical separation. The two types of antennas, for horisontal and for vertical polarization, are also shown.

The attenuation length for RF signals in the ice varies with depth due to its temperature dependence. The cold ice at the top has the best propagation properties with attenuation lengths better than $1.5 \mathrm{~km}$; a representative mean for the full depth is $\sim 800 \mathrm{~m}$ (@300 MHz). As the ice at the top gradually change into firn the index of refraction varies in the top part of the glacier. This causes bending and reflection of the radio signals and consequently the part of the ice that is visible from one antenna depends on the installation depth. Simulation has shown that the effective volume is two times larger for antennas at $200 \mathrm{~m}$ depth than for a surface installed antenna. Including drilling considerations $200 \mathrm{~m}$ was chosen as the design depth. Simulation studies show that smaller spacing than $2 \mathrm{~km}$ is sub-optimal as volumes seen by different stations start to overlap. The ARA design array is simulated to have an aperture of $\sim 300 \mathrm{~km}^{3} \mathrm{sr}$ at $\mathrm{E}_{\mathrm{v}} \sim 10^{18} \mathrm{eV}$. The angular region covered is from $\sim 45^{\circ}$ above the horizon down to the horizon where the neutrino visibility is limited by energy dependent absorption in the Earth [14].

The ARA collaboration has recently reported on a full analysis of $330 \mathrm{M}$ events recorded during 224 days with the 'Testbed' system [15]. The time of the 'South Pole Summer Work Period' was excluded due to increased electronic noise. Three different analysis schemes were tested. Timing differences in the received signal allowed to determine the direction of the radiation and to exclude events in directions where repeating events was found. The cause of all repeating events is not clear, but frequency dependent criteria should be possible to apply in later analysis. The direction to the South Pole Station was excluded. Further cuts were used on the received signal amplitude variation, electronics saturation, anthropogenic noise and signal shape compatibility with expectations for neutrino events. No events survived all cuts, yielding a limit on the neutrino flux and more importantly demonstrated to first order a working detector concept. This allows projecting to the sensitivity for a future, full 37 station ARA system, fig. 7.

\subsection{ARIANNA}

The Antarctic Ross Ice-shelf ANtenna Neutrino Array (ARIANNA) is a proposed array located in Moore's Bay on the immense Ross ice-shelf at a distance of $110 \mathrm{~km}$ from the US Antarctic McMurdo Station. As the $\sim 600$ m thick ice is floating on sea-water the downgoing RF 


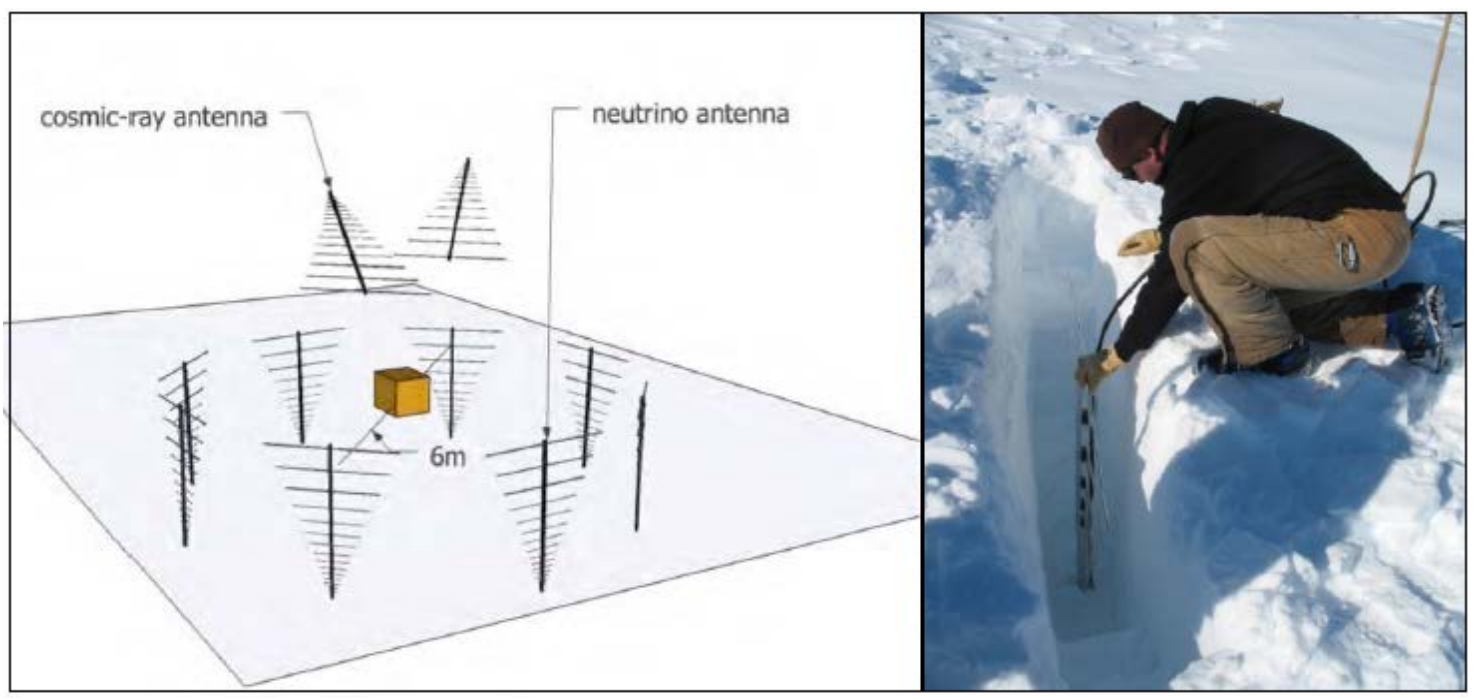

Fig. 6. The ARIANNA project. Left: Schematic drawing of one of the proposed 1236 ARIANNA stations showing the 8 downward oriented LPDA antennas for neutrinos and two upward directed antennas for cosmic rays used for calibration and background suppression. The diameter of the octagon is $12 \mathrm{~m}$, the spacing between stations is $1 \mathrm{~km}$. The DAQ electronics is contained in the central box. Power and communication equipment is not shown [17]. Right: Installation of a LPDA antenna just below the surface, in a hole dug in the firn. The hole is then back-filled with firn.

radiation will be reflected up. This increases the sensitivity of ARIANNA which thereby can see events both in the direct radiation and in the reflected radiation.

Present plans for ARIANNA calls for 36*36 stations in an array with $1 \mathrm{~km}$ station spacing. Each station would consist of eight down-looking antennas installed in the firn, just below surface, see fig. 6. No drilling is needed. Two upward looking antennas would serve to detect incoming cosmic rays to allow calibration and suppresion of possible backgrond from these. The antennas used are Log-Periodic Dipole Array (LPDA) antennas with bandwidth 100 - 1300 MHz. These antennas are linearly polarized. They are installed into holes dug in the firn to a depth of $\sim 1 \mathrm{~m}$. Their size and geometry is not limited by deployment requirements. Each station will have local power from solar panels (possibly a wind-turbine and battery back-up) for the electronics and for communication over WiFi or an Iridium link. The site is screened from the McMurdo station by Minna Bluff and essentailly free from antropogenic radio-noise. A WiFirepeater station is installed at the mountain and allows for high speed communication with the array from the station [16][17].

ARIANNA has installed smaller test stations in a project dubbed Hexagonal Radio Array (HRA). Each HRA station has 4 recieveer antennas (instead of 8+2). Three HRA stations were installed in 2012, the remaining 4 in the 2014-15 season. Improvments of the electronics and the trigger system was done after the initial installation, implementing e.g. a pattern trigger allowing for a low $\left(<4 * \sigma_{\mathrm{v} \text {-therm }}\right)$ trigger threshold combining $\mathrm{mHz}$ rates with high sensitivity. The DAQ can handle rates of $100 \mathrm{~Hz}$, but this would result in large data volumes to transfer, without increased sensitivity. The amplifier is low noise with a smooth attenuation for high amplitude input signals to yield useful data also for large signals. A calibration pulser system with a horisontal antenna installed just below the snow surface at $\sim 20 \mathrm{~m}$ distance makes operation verification and monitoring possible. The total power used per station is $\sim 7 \mathrm{~W}$ (possible to reduce by limiting communication).

Extensive tests of the attenuation and reflection properties of the ice have been made [18]. The attenuation length is $\sim 400-500 \mathrm{~m}$ as averaged over the ice thickness and the reflection coefficient at the interface to the sea-water corresponds to the theoretical expectation of $\sim 0.82$. 

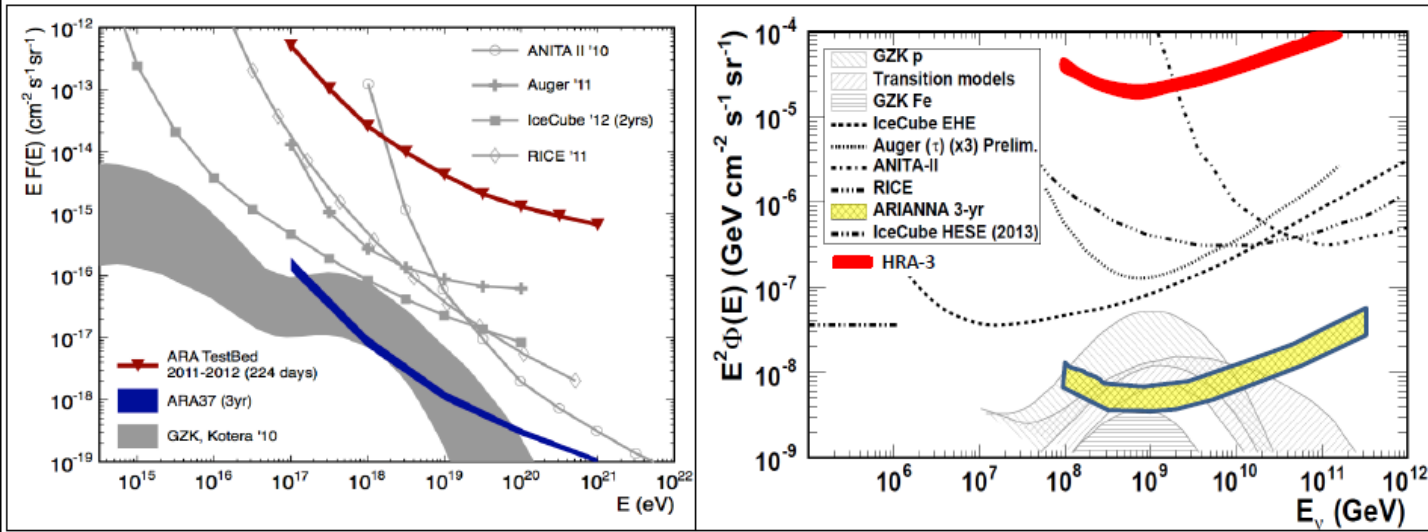

Fig. 7. Left: The ARA Testbed limit (red line) and the projected 3 year sensitivity of the propsed full ARA37 system (blue line) [15]. Right: The ARIANNA HRA-3 limit (red line) and projected 3 year sensitivity of the proposed full ARIANNA system. Both plots show the range of popular models, in the right panel some more pessimistic models are added. Note that the flux in the left plot is multiplied by E and in the right plot by E2 [17] (The HRA-3 limit is added to fig. 6 in [17]).

This lower ice surface is flat to the level detected through extensive bounce tests. Thus no adverse effects are expected due to image distortions.

Data from the three first HRA stations have been analysed and used for a first perfomance test limit [17]. The data was taken January-April 2014 and comprize 170 live station-days. The trigger rate was dominated by thermal events, thus the trigger rate followed the temperature. No particular measure was needed to remove external antropogenic noise. Three selection criteria were used. A self-correlation check where a time shifted replica of the recorded waveform is correlated to the original waveform. Thermal noise has a strong correlation only at no timeshift, while a real signal would show correlation also at a different time shift. The second test investigate the frequency content. Most remaining noise, e.g. from the station iteslf, has a narrow frequency peak while neutrino events would have power over a braoder frequency band. The third requirement compares the observed waveform to a library of waveforms from simulations corrsponding to possible neutrino event variations [19]. In this test the noise events (from a clock trigger) show low correlation and can be removed. Windy conditions, and in particular around a time when a wind generator broke down, showed increased correlation but still far from the main signal region for neutrino events. No event survived this set of three conditions and a limit was obtained. This limit is comparable to the limit from the ARA Testbed. Note that the conditions in number of stations, live-time etc are different so the equality of the limits in itself is not very meaningful. The conclusion is that both ARA and ARIANNA have demonstrated that noise and background events can be eliminated at a corresponding level in exposure.

The proposed full ARIANNA station would have improved performace due to the larger number of antennas and the upward pointing antennas. Local computing power may eliminate most noise events through the first of the cuts and also provide a first analysis of remaining neutrino candidate events. A flash memory of $32 \mathrm{~Gb} /$ station is sufficient for storage of one year of data in case the comunication link is broken or if power consumption needs to be reduced.

The ARIANNA system would cover the full angular region from zenith to somewhat below horizon where the neutrinos are absorbed by the Earth giving an energy dependent angular cut-off. Most of this range, in particular the directions closer to vertical, would be seen through the RF signals reflected at the ice-sea-water interface. As the latitudeof the site is $\sim 78^{\circ} \mathrm{S}$ together with the rotation of the Earth a somewhat extended region of the sky will covered and eventual neutrino sources would be observed at varying altitudes. The aperture has been 
calculated by simulation and reaches $\sim 10^{3} \mathrm{~km}^{3} \mathrm{sr}$ at $10^{18} \mathrm{eV}$. The relative energy resolution $\sigma_{\mathrm{E}} / \mathrm{E}$ is expected to be in the range 2.5-5 and the uncertainty largely depends on the observation angle relative to the Cherenkov angle (where the peak intensity is). As the Cherenkov radiation is polarized the direction of the incoming neutrino can be resolved to $2.5^{\circ}-3^{\circ}$ by using the power ratio in antennas with different orientations. The two upward antennas would help to remove rare background from cosmic rays, a conservative estimate gives 0.2 events/ year remaining in the full system. A large sample of CR events will help in system calibration and verification. The sensitivity to GZK flux is shown in fig 7. Only the most pessimistic models predict fluxes to low for event detection, most current GZK models would yield events, albeit there are large variation in the number of events predicted. The large variation implies that experimental results will be able to discriminate between the different models for the highest energy cosmic rays.

\subsection{GNO}

The Greenland Neutrino Observatory (GNO) is the most recent addition to the projects aiming for neutrino detection with radio technique in ice [20]. The location is at the Summit Station on Greenland, latitude $72^{\circ} \mathrm{N}$, where the ice is $3 \mathrm{~km}$ thick and there is a layer of water below the ice. The RF absorption has been measured by bouncing radio pulses at the bottom of the ice and found to be $>1 \mathrm{~km}$ in the upper half of the ice and dropping to $\sim 500 \mathrm{~m}$ at the bottom of the ice as it gradually becomes warmer. GNO reciever antennas are to be installed in drilled holes, but due to a different firn density gradient, the depth needed is $100 \mathrm{~m}$ (cf $200 \mathrm{~m}$ for ARA). The first installation of RF equipment for testing of the suitability of the site and test of antennas etc will be done in the summer of 2015. These recievers are planned for surface deployment. Very conviniently two open deep drilled holes exist at the site (from the DISC and GISP2 projects) and may be used for calibration pulsers and other tests. If the site tests are succesful GNO will offer a view of the sky which is complementary to the two projects in Antarctica. Partial overlap of the observed sky in a band around horizon $\left(\sim 18^{\circ}\right.$ wide with ARA, $\sim 30^{\circ}$ wide with ARIANNA) will provide important possibilities for cross-checks of the observations of GZK neutrinos, in particular if point sources or emission regions are found in the overlapped part of the sky.

\section{Summary and Conclusion}

The enigma of the extremly high energy cosmic rays with energies of $10^{20} \mathrm{eV}$ needs to be solved. A search for neutrinos with energies $>10^{17} \mathrm{eV}$ and observational determination of their spectral and directional properties will provide key information on the high energy universe. Simultaneously properties of neutrino interactions may reveal new physics. This requires monitoring large quantitites of target matter, at the scale of $\sim 100-1000 \mathrm{~km}^{3}$. This can be achieved most efficiently by installing radio detection stations in natural ice. Three projects for in ice radio detection of neutrinos, ARA, ARIANNA and GNO are suggested and are in different phases of promising prototype work. Two of the projects have already run small test setups and demonstrated performance by succesfully analysing their first test data. Let’s build them!

\section{Acknowledgments}

The author thanks in particular M. DuVernois of the ARA collaboration, S.W. Barwick and C. Reed of the ARIANNA collaboration and A. Vieregg of the GNO collaboration for pointing to the most recent information on the projects. 


\section{References}

[1] IceCube Collaboration, Evidence for High-Energy Extraterrestrial Neutrinos at the IceCube Detector, Science (342) 1242856 (2013) [arxiv1311.5238].

[2] IceCube Collaboration, Observation of High-Energy Astrophysical Neutrinos in Three Years of IceCube Data, Phys. Rev. Lett. 113, 101101 (2014), [arxiv1405.5303].

[3] IceCube Collaboration, IceCube-Gen2: A vision for the Future of Neutrino Astronomy in Antarctica, [arxiv1412.5106].

[4] J. Beringer et al. (PDG) PR D 86, 010001 (2012), http://pdg.lbl.gov/2012/reviews/rpp2012-revcosmic-rays.pdf .

[5] K. Greisen, Phys. Rev. Lett.16, 748 (1966); G.T. Zatsepin and V. A. Kuzmin, JETP Lett.4, 78 (1966).

[6] S.R. Klein, A. Connolly, Neutrino Absorption in the Earth, Neutrino Cross-Sections, and New Physics, [arxiv 1304.4891]

[7] R. Engel, D. Seckel, T. Stanev, Neutrinos from propagation of ultrahigh energy protons, Phys. Rev. D, 64 093010, (2001).

[8] G.A. Askaryan, Excess negative charge of an electron-photon shower, JETP 14, 441 (1962);

G.A. Askaryan, Coherent radio emission from cosmic showers in air and in dense media, JETP 21658 (1965).

[9] J. Alvarez-Muniz, E. Zas, The LPM effect for EeV hadronic showers in ice: Implications for radio detection of neutrinos. Phys. Lett. B434, 396 (1998).

J. Alvarez-Muniz, A. Romero-Wolf, E. Zas, Cherenkov radio pulses from electromagnetic showers in the time domain. Phys. Rev. D 81, 123009 (2010).

[10] P. Gorham et al. (ANITA Collaboration), Observations of the Askaryan Effect in Ice, Phys. Rev. Lett. 99, 171101 (2007).

[11] I. Kravchenko et al. Performance and simulation of the RICE detector. Astroparticle Phys. 19, 15 (2003)

[12] I. Kravchenko et al. RICE limits on the diffuse ultrahigh energy neutrino flux, Phys. Rev. D 73 082002 (2006)

[13] I. Kravchenko et al. Status of Rice and preparations for the next generation neutrino experiment in Antarctica. NIM A692 233 (2012)

[14] P. Allison et al. Design and initial performance of the Askaryan Radio Array prototype EeV neutrino detector at the South Pole, Astroparticle Phys. 35, 457 (2012).

[15] P. Allison et al. First Constraints on the Ultra-High Energy Neutrino Flux from a Prototype Station of the Askaryan Radio Array, [arxiv:1404.5285], Astroparticle Phys. 7062 (2015)

[16] S.W. Barwick et al., Design and performance of the ARIANNA Hexagonal Radio Array Systems, [arxiv:1410.7369], Submitted to IEEE TNS.

[17] S.W. Barwick et al., A First Search for Cosmogenic Neutrinos with the ARIANNA Hexagonal Radio Array, [arxiv:1410.7352], Astroparticle Phys. 7012 (2015)

[18] J.C. Hansson et al., Radar Absorption, Basal Reflection, Thickness, and polarization Measurements from the Ross Ice Shelf, Journal of Glaciology 61(227) 438 (2015), [arxiv:1410.7134]

[19] S.W. Barwick, Time-domain response of the ARIANNA detector, Astroparticle Physics 62139 (2015)

[20] J. Avva et al., An in situ measurement of the radio-frequency attenuation in ice at Summit Station, Greenland, [arxiv:1409.5413], Submitted to Journal of Glaciology. 